\title{
Kindeswohl in der Krise?
}

\section{Zum professionellen Handeln im organisierten Kinderschutz}

Mit der Neu-Programmierung der Kinder- und Jugendhilfe durch den in Kraft getretenen §8a SGB VIII und dem Bundeskinderschutzgesetz wird professionelles Handeln in der Krisendiagnose von Kindeswohlgefährdung immer wieder neu herausgefordert. Der Beitrag fokussiert Schutzlogiken, die sich am Instrument des Schutzplans zeigen lassen.

$\mathrm{D}$ er Verdacht auf Krisen ${ }^{1}$ des Aufwachsens stellt die sozialpädagogische Professionalität in zunehmendem Maße vor die Herausforderung, Gefährdungslagen von Kindern und Jugendlichen einzuschätzen. Damit wird vermehrt die Anforderung an professionelles Handeln gestellt, Entscheidungen ${ }^{2}$ vor dem Hintergrund der Unbestimmtheit von Kindeswohl zu treffen.

Eine Fokussierung des Kinderschutzes in der Kinderund Jugendhilfe lässt sich anhand der jährlich ansteigenden Fallzahlen interpretieren. Im Jahr 2019 wurden, seit Beginn der statistischen Erfassung im Jahr 2012, die höchsten jährlichen Fallzahlen mit 173.029 Gefährdungseinschätzungen von Jugendämtern gem. \$8a SGB VIII gemessen. Von 2012 bis 2017 konnte im Durchschnitt eine jährliche Zuwachsrate von sechs Prozent festgestellt werden. Im Anschluss daran sind die Gefährdungseinschätzungen ab 2017/18 nochmals auf durchschnittlich zehn Prozent angestiegen, was auf die öffentliche Diskussion von Kinderschutzverläufen, die veränderten Kooperationsstrukturen mit anderen Institutionen und eine erhöhte Aufmerksamkeit in den Verfahren und Organisationsstrukturen des institutionel-

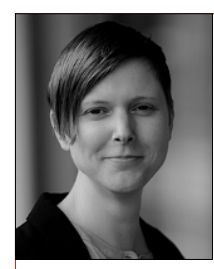

\section{Nina Kläsener}

Universität Bielefeld, Bielefeld, Deutschland

*1979; Diplom-Pädagogin, langjährige Praxiserfahrung in der freien und öffentlichen Kinder- und Jugendhilfe. Seit 2018 wissenschaftliche Angestellte in der AG 8 Soziale Arbeit der Fakultät für Erziehungswissenschaft an der Universität Bielefeld. nina.klaesener@uni-bielefeld.de

Zusammenfassung Der Verdacht auf Kindeswohlgefährdung stellt die sozialpädagogische Professionalität in zunehmendem Maße vor die Herausforderung, Gefährdungslagen von Kindern und Jugendlichen einzuschätzen und Entscheidungen zu treffen. Der Beitrag diskutiert Entscheidungslogiken am Instrument des Schutzplans.

Schlüsselwörter Krise, Kinderschutz, Kindeswohl, ASD, Schutzplan, Professionelles Handeln, Organisation len Kinderschutzes zurückgeführt wird (KomDat 2020). Die steigende Entwicklung der Fallzahlen nach $\$ 8$ a SGB VIII hat sich im Rahmen der Zusatzerhebung in der Corona-Pandemie im Jahr 2020 jedoch vorerst nicht fortgesetzt, was auf die nicht präsenten Hinweisgeber, wie Kitas und Schulen, zurückgeführt wird. Ein Teil der Jugendämter befürchtet zudem, dass trotz der weiteren Priorisierung des Kinderschutzes in der Corona-Krise das Dunkelfeld gewachsen ist (Mairhofer et al. 2020). Demnach weisen die institutionell festgestellten Gefährdungslagen vielmehr auf administrative Artefakte hin, die institutionelle Tatsachen schaffen. Über die Grundgesamtheit der Gefährdungsfälle geben sie jedoch keinen Aufschluss.

Die Kinderschutzfokussierung in der Kinder- und Jugendhilfe wurde durch den 2005 in Kraft getretenen Schutzauftrag bei Kindeswohlgefährdung nach \$8a SGB VIII und nicht zuletzt durch die Regelungen des Bundeskinderschutzgesetzes verdichtet, wodurch professionelles Handeln im Kinderschutz gesetzlich zur Sicherstellung von Kindeswohl gerahmt wurde. Durch die Ausrichtung des Kinder- und Jugendhilfesystems auf den Kinderschutz wird befürchtet, dass sich die Kinder- und Jugendhilfe in Richtung einer Kindeswohlgefährdungsvermeidungsstrategie (Dahmen und Kläsener 2019) verschiebt, insofern, als sich die Leistungserbringung weniger am Bedarf als an der diagnostischen Kategorie „Kindeswohlgefährdung“ orientiert.

\section{Kindeswohl als Maßstab zur Krisenbestimmung}

Das professionelle Handeln richtet sich im Feld des Kinderschutzes an der Deutung des Begriffs Kindeswohl aus (Ziegler 2020), der als Kontingenzformel in der Sozialen Arbeit das Dreiecksverhältnis von Kind, Eltern und Staat bestimmt. Dem Wohl des Kindes kommt hierbei eine Zielgröße in der Vermittlung vom Kindesund Elternrecht zu. Im Schutzauftrag nach $\$ 8$ a SGB VIII wird professionelles Handeln zur Abwendung der Gefährdung des Kindeswohls überführt. Die von Fachkräften festzustellende Krisendiagnose „Kindeswohl- 
gefährdung“ bedeutet, „dass die Rede von einer Krise Zustimmung zu mobilisieren sucht für eine spezifische Situationsdefinition und/oder Interventionsplanung durch einen Appell an gemeinsame Interpretation gesellschaftlicher Wirklichkeit“ (Dollinger 2004, S. 383). Die Deutung von Kindeswohl bzw. der Verdacht auf Gefährdung werden in der Nutzung der diagnostischen Instrumente auf der Ebene der Fachkräfte als Krisenbestimmung wirksam. Dies birgt die Gefahr, Adressat innen bei der Bestimmung von Krisenlagen nicht zu beteiligen und durch Risikoeinschätzungsinstrumente Entscheidungen vorzustrukturieren (Ackermann 2021). Standardisierungsverfahren dienen dabei als Bestandteile organisationaler Entscheidungsarchitekturen (Büchner 2017) und beeinflussen damit das professionelle Handeln der Fachkräfte. Durch die StandardisierungsEffekte wird allerdings eine Engführung professioneller Handlungsmöglichkeiten ins Feld geführt, so dass Tendenzen von „Deprofessionalisierung “ (Bastian 2017) im institutionellen Kinderschutz zu befürchten sind.

\section{Zum Verhältnis von Profession und Organisation im Kinderschutz}

Strukturtheoretische Fragen schließen an die Diskussion um Professionalisierung im Kinderschutz aufgrund der Nicht-Standardisierbarkeit Sozialer Arbeit an, da diese im Kern als professionalisierungsbedürftig gilt (Oevermann 2000). Oevermann sieht hier allerdings nicht nur aufgrund „der Übernahme gesellschaftlicher Kontrollaufgaben im Kontext der Aufrechterhaltung von Recht und Gerechtigkeit, sondern auch aufgrund ihrer Einbettung in bürokratische Organisationen " (Hollenstein 2018, S. 6) Grenzen der Professionalisierbarkeit.

Die strukturtheoretische Position mit der Perspektive des Arbeitsbündnisses als „Kern-Modell-professionalisierten Handelns“ (Oevermann 1996, S. 115) begnügt sich mit der Antwort, dass durch das widersprüchlich angelegte „Strukturdilemma“ (ebd., S. 71) in der Adressierung von gleichzeitiger Hilfe und Kontrolle, ein Arbeitsbündnis nicht zu schließen sei, denn „eine Professionalisierung von Hilfe [...] lässt sich mit der Professionalisierung von Kontrolle [...] nicht unter einen Hut bringen“ (ebd., S. 139). Dies wirft im Anschluss an das strukturelle Dilemma die Frage nach der grundsätzlichen Erfüllung des Schutzauftrags auf, insofern, als der Professionalisierung der immanente und gleichzeitige Auftrag von Hilfe und Kontrolle zur Herstellung von Kindeswohl entgegenstehe.

Die damit angesprochenen Anforderungen des immanenten Doppelauftrags professioneller Praxis wirken sich auch auf Organisationsstrukturen von Jugendäm- tern aus. Institutionelle Logiken scheinen diese Anforderungen durch eine strukturelle Trennung von Hilfe- und Kontrollaspekten, in Abteilungen zur Einschätzung von Gefährdungslagen und Bereichen in der Zuständigkeit von Hilfen zur Erziehung aufzulösen. Hierbei stellt sich die Frage nach dem grundsätzlichen Verhältnis und der Verschränkung von fachlichem Handeln und formalen Organisationsbedingungen und der Bestimmung von Professionalität in der Sozialen Arbeit unter bestimmten „Kontextbedingungen“ (Nadai und Sommerfeld 2005, S. 181). Demnach können Organisationskontexte professionelles Handeln ermöglichen oder aber auch erschweren. ${ }^{3}$ Die These des grundsätzlichen Konfliktes zwischen bürokratischer und professioneller Logik in Organisationen konnte mittlerweile empirisch widerlegt werden, so dass „bürokratische und professionelle Elemente durchaus strukturell kombinierbar sind“ (Klatetzki und Tacke 2005, S. 14) und eher von einer Verschränkung derselben auszugehen sei. Professionelle Organisationen „weisen lediglich die Besonderheit auf, dass die Technologien im wissenschaftlich ausgebildeten Professionellen verortet sind“ (Klatetzki 2005, S. 254). Wesentlich ist demnach, dass die Bedingungen von Organisationen auf fachliche Positionierungen Einfluss nehmen. Ebenfalls kann jedoch davon ausgegangen werden, dass die kollektiven Deutungsmuster von Fachkräften wiederum die Organisation bedingen. Organisationskontexte können daher als sich verschränkende Aushandlungsordnungen (Nadai und Sommerfeld 2005) verstanden werden, in denen professionelles Handeln und damit Schutzlogiken hergestellt und reproduziert werden.

Im Medium des Schutzplans wird die situierte Aushandlung der Bedingungen von professionellem und organisationalem Handeln deutlich, was im Folgenden auf der Grundlage eigener Untersuchungen zu Entscheidungsrationalitäten im Kinderschutz aufgezeigt wird.

\section{Schutzplan als Instrument zur Krisenverhandlung im organisierten Kinderschutz}

Vielfach haben sich bei einem Verdacht auf Kindeswohlgefährdung in den Jugendämtern sogenannte "Schutzpläne“ etabliert. Sie sollen die Abwendung der Gefährdung sicherstellen, wenn das Kind in der Familie verbleibt, meist durch schriftlich fixierte Vereinbarungen zwischen Eltern, Jugendamt und Akteur_innen der Hilfen zur Erziehung. Die in der Praxis sehr gebräuchlichen Schutzpläne (oder auch Schutzkonzepte genannt) haben keine gesetzliche Grundlage, sind bisher theoretisch wenig bestimmt und ihre Handhabung wurde bislang kaum - mit Ausnahme der Studie von Lenkenhoff 
et al. (2013) zu Schutzkonzepten in der Hilfeplanung untersucht.

Das Instrument des Schutzplans lässt sich vor dem Hintergrund der bisherigen Ausführungen in Bezug auf folgende Funktionsverschränkungen in der Krisenverhandlung deuten:

1. Professionelles Handeln als Krisendiagnose;

2. Bedingungen von professionellem und organisationalem Handeln;

3. Kontraktlogik professionellen Handelns.

Im Rahmen der Krisendiagnose ${ }^{4}$ kommt der Schutzplanung im Falleingang eine besondere Bedeutung zu, da hier noch vor dem Hausbesuch gemäß \$8a SGB VIII die Gefährdungslage im Zusammenwirken mehrerer Fachkräfte eingeschätzt wird. In der Deutung der Gefährdungslage wird bereits über die Notwendigkeit von Schutzplänen beraten, ohne zwingend schon einen Kontakt zur Familie hergestellt zu haben, was die Bestimmung der im Schutzplan relevanten Inhalte noch vor der Aushandlung mit der Familie vorstrukturiert. Der Vorab-Verdacht einer potenziellen Krise in Bezug auf eine mögliche Kindeswohlgefährdung löst damit den sozialpädagogischen Handlungsmodus bereits aus und fordert eine Entscheidung.

Anders verhält es sich bei bereits installierten Hilfen zur Erziehung. Hier tritt bei der Einschätzung und Bewertung von ,Kindeswohlgefährdungen“ häufig neben den Hilfeplan ein Schutzplan, der die Maßnahmen über professionelles Handeln von Fachkräften faktisch entscheidet und in der Regel konkrete Anforderungen stellt, die als verpflichtende ,Auflagen' formuliert werden. Lenkenhoff et al. (2013, S. 15) sprechen davon, dass bislang an „keiner Stelle - weder rechtlich noch fachlich - durchdekliniert [... sei, wie] Schutzkonzepte rechtsstaatlich und fachlich korrekt ausgestaltet werden [können]“. Im Schutzplan zeigen sich potenziell hoheitsstaatliche Kontrollaufgaben, die eigentlich den Familiengerichten nach $\mathbb{} 1666$ BGB vorbehalten sind. Diese scheinen sich auf die Ebene der Jugendämter und ihrer dienstleistungsorientierten Ausrichtung als „Soziale Dienste" zu verlagern.

Da professionelle Handlungen stets in Organisationen wirksam werden, ist der Einfluss von Organisationsstrukturen wesentlich. Deshalb kann von einer gleichzeitigen Bedingung von professionellem und organisationalem Handeln in der Herstellung des Schutzplans ausgegangen werden. Zum einen werden professionelle Positionierungen durch Organisationskulturen beeinflusst, mit welchen Inhalten ein Schutzplan ausgestattet wird. Zum anderen nehmen die professionellen Handlungen auf Organisationsformen Einfluss und le- gen Verfahrensstandards fest, in welcher Fallkonstellation Schutzpläne zum Einsatz kommen. Der Landschaftsverband Rheinland (LVR) empfiehlt beispielsweise in seiner Orientierungshilfe zu Schutzplänen, dass „Inhalt des Schutzplans [...] dabei die auf die Gefährdung bezogenen zwingend notwendigen Maßnahmen und deren Kontrolle [sind], während im Hilfeplan weitergehende (freiwillige) Ziele zur Verbesserung der Erziehungssituation formuliert werden, die zwar hilfreich, aber nicht unabdingbar notwendig sind." (LVR 2015, S. 27). Die funktionale Trennung der Dokumente in Hilfe- und Kontrollmodalitäten dient dementsprechend der Legitimation von Verfahrensstandards innerhalb der Organisation. Somit kann der Schutzplan als aktenförmiger Nachweis innerhalb der Organisation genutzt werden. Dies ermöglicht die Absicherung von Fachkräften und eine zukünftige Gerichtsbarkeit.

Aufgrund der Kontraktlogik des Schutzplans besteht die Gefahr, dass alleine professionelle Perspektiven zur Gefahrenabwehr sichtbar werden und Adressat_innen lediglich in der zu leistenden Unterschrift im Dokument beteiligt werden. Im Modus „stellvertretender Krisenbewältigung“ (Oevermann 2013, S. 120) kommt der professionellen Deutung damit eine höhere Rationalität zu, die eine adressat_innenorientierte Aushandlung von Gefährdungsinhalten und damit vor allem Hilfemöglichkeiten verhindert. Eine einseitige Fokussierung auf Kontrollelemente fördert eine Passivierung von Adressat_innen, die lediglich im Zuge der Einhaltung von Schutzmaßnahmen in Aktivität umgekehrt werden soll. Dabei gerät leicht aus dem Blick, wie Gefahrenabwehr und Hilfeangebote in der gleichzeitigen Bestimmung miteinander verbunden werden können.

In Anlehnung an partizipative Forschungsansätze (Bergold und Thomas 2012), besteht das Potenzial, Adressat_innen nicht lediglich krisenhaft in der Gefahrenabwehr zu berücksichtigen, sondern am Schutzauftrag teilhaben zu lassen und das als Ausgangspunkt einer partizipativen Professionalität innerhalb von Organisationen $\mathrm{zu}$ verstehen. Organisationskontexten kommt hierbei die Aufgabe als Aushandlungsort zu, Professionalität zu ermöglichen. Im Kinderschutz wird demnach nicht nur auf Krisen reagiert, sondern professionelles Handeln markiert hier, was eine Krise ist und wird auch selbst aufgrund von Organisationsbedingungen als krisenhaft erlebt.

Eingegangen. 19. März 2021

Angenommen. 28. April 2021 
Funding. Open Access funding enabled and organized by Projekt DEAL.

Open Access. Dieser Artikel wird unter der Creative Commons Namensnennung 4.0 International Lizenz veröffentlicht, welche die Nutzung, Vervielfältigung, Bearbeitung, Verbreitung und Wiedergabe in jeglichem Medium und Format erlaubt, sofern Sie den/die ursprünglichen Autor(en) und die Quelle ordnungsgemäß nennen, einen Link zur Creative Commons Lizenz beifügen und angeben, ob Änderungen vorgenommen wurden.

Die in diesem Artikel enthaltenen Bilder und sonstiges Drittmaterial unterliegen ebenfalls der genannten Creative Commons Lizenz, sofern sich aus der Abbildungslegende nichts anderes ergibt. Sofern das betreffende Material nicht unter der genannten Creative Commons Lizenz steht und die betreffende Handlung nicht nach gesetzlichen Vorschriften erlaubt ist, ist für die oben aufgeführten Weiterverwendungen des Materials die Einwilligung des jeweiligen Rechteinhabers einzuholen.

Weitere Details zur Lizenz entnehmen Sie bitte der Lizenzinformation auf http://creativecommons.org/licenses/by/4.0/deed.de.

1. Im Beitrag wird das Krisenverständnis von Dollinger geteilt, da „weder Krisen noch pädagogisches Wissen [...] folglich konstant zu setzen [sind], sondern es ist ein dynamisches Verhältnis der sozialen Realität, des pädagogischen Wissens und ihrer Relation zueinander anzunehmen; sie setzen sich gegenseitig in Bezug, objektivieren ,richtige` Lösungen für angemahnte Problemlagen und definieren pädagogische Handlungsfelder" (Dollinger 2006, S. 18).

2. Nach $\int 8$ a SGB VIII heißt es im Wortlaut: „[...] das Gefährdungsrisiko im Zusammenwirken mehrerer Fachkräfte einzuschätzen". ( $\$ 8$ a SGB VIII) Im Beitrag wird in der weiteren Argumentation von dem Begriff der Entscheidung gesprochen, da auch bei der Einschätzung von Gefährdungslagen der weitere Fallverlauf prozessiert wird, was auf Entscheidungshandeln zurückzuführen ist.

3. Eine „Fallzablenbegrenzung " in den Jugendämtern wird bundesweit diskutiert (Bundesarbeitsgemeinschaft ASD 2020). In der Steuerungsverantwortung der kommunalen Kinder-und Jugendhilfe entscheiden Jugendämter selbst über die Fallzablenauslastung der Mitarbeitenden, was sich auf die Organisationsstruktur und das professionelle Handeln auswirkt. Hierbei muss zudem angemerkt werden, dass jugendamtliche Verfahren im Rabmen kommunaler Selbstverwaltung festlegen, wann ein Fall zum Fall wird und damit im Rahmen der Fallzablen berücksichtigt wird. Dies zeigt Auswirkungen auf die Bedingungen der Fallbearbeitung.

4. Der Begriff „Krisendiagnose“ kategorisiert vermeintlich objektive Kriterien, „da Krisendiagnosen stets Normalität definieren und Wertungen über sie mitteilen, sind sie niemals neutrale Darstellungen eines Zustandes oder Prozesses, sondern verweisen auf Definitionsmacht." (Dollinger 2004, S. 381).

\section{Literatur}

Ackermann, T. (2021). Risikoeinschätzungsinstrumente und professionelles Handeln im Kinderschutz. Sozial Extra, 45(1), 42-48.

Bastian, P. (2017). Negotiations with a risk assessment tool. Standardized decision-making in the United States and the deprofessionalization thesis. Transnational Social Review, 7(2), 206-218.

Bergold, J., \& Thomas, S. (2012). Participatory research methods: a methodological approach in motion [110 paragraphs]. Forum Qualitative Sozialforschung/ Forum: Qualitative Social Research, 13(1), Art. 30. http:// nbn-resolving.de/urn:nbn:de:0114-fqs1201302. Zugegriffen: 29.03.2021.
Büchner, S. (2017). Der organisierte Fall: Zur Strukturierung von Fallbearbeitung durch Organisation. Wiesbaden: Springer VS.

Bundesarbeitsgemeinschaft ASD (2020). Newsletter BAG ASD, ASD Report 2020-1. https://www.bag-asd.de/wp-content/uploads/2020/01/ASDReport-1-2020.pdf. Zugegriffen: 8. März 2021.

Dahmen, S., \& Kläsener, N. (2019). Kinder- und Jugendhilfe als Kindeswohlgefährdungsvermeidungsstrategie? Soziale Passagen, 10(2), 197-210.

Dollinger, B. (2004). Krisenintervention als Aufgabe der Sozialen Arbeit. Anmerkungen zu einer sozialpädagogischen Krisentheorie. Zeitschrift für Sozialpädagogik, 2, 377-396.

Dollinger, B. (2006). Die Pädagogik der Sozialen Frage. (Sozial-)Pädagogische Theorie vom Beginn des 19. Jahrhunderts bis zum Ende der Weimarer Republik. Wiesbaden: Springer VS.

Hollenstein, L. (2018). Gesellschaft, Organisation, Professionalität-Zur Relevanz von Professionspolitik in der Sozialen Arbeit. Wiesbaden: Springer VS.

Klatetzki, T. (2005). Professionelle Arbeit und kollegiale Organisation. Eine symbolisch interpretative Perspektive. In T. Klatetzki \& V. Tacke (Hrsg.), Organisation und Profession (S. 253-283). Wiesbaden: VS.

Klatetzki, T., \& Tacke, V. (2005). Organisation und Profession. Wiesbaden: VS.

KomDat Jugendhilfe (2020). Heft 2 \& 3. http://www.akjstat.tu-dortmund.de/fileadmin/user_upload/2020_Heft_02-03_KomDat.pdf. Zugegriffen: 29. März 2021.

Landesjugendamt Rheinland (2015). Gelingensfaktoren bei der Wahrnehmung des Schutzauftrages gemäß $\int 8 a$ SGB VIII. Köln: LVR.

Lenkenhoff, M., Adams, C., Knapp, H., \& Schone, R. (2013). Schutzkonzepte in der Hilfeplanung. Eine qualitative Untersuchung zur Funktion und zur Wirkungsweise von Schutzkonzepten im Rahmen ambulanter Erziehungshilfen. LWL-Landesjugendamt Westfalen, Ideen und Konzepte. https://www.lwl-landesjugendamt.de/media/filer_public/78/3c/783ca5e2817f-4c23-a240-9d05b3af7cfb/abschlussbricht_schutzkonzepte_2.pdf. Zugegriffen: 24. Febr. 2021.

Mairhofer, A., Peucker, C., Pluto, L., van Santen, E., \& Seckinger, M. (2020). Kinder- und Jugendhilfe in Zeiten der Corona-Pandemie. DJIJugendhilfeb@rometer bei Jugendämtern. München. https://www.dji.de/ fileadmin/user_upload/bibs2020/1234_DJI-Jugendhilfebarometer_Corona. pdf. Zugegriffen: 24. Febr. 2021.

Nadai, E., \& Sommerfeld, P. (2005). Professionelles Handeln in Organisationen - Inszenierungen der Sozialen Arbeit. In M. Pfadenhauer (Hrsg.), Professionelles Handeln (S. 181-207). Wiesbaden: Springer VS.

Oevermann, U. (1996). Theoretische Skizze einer revidierten Theorie professionalisierten Handelns. In A. Combe \& W. Helsper (Hrsg.), Pädagogische Professionalität. Untersuchungen zum Typus pädagogischen Handelns (S. 70-182). Frankfurt a. M.: Suhrkamp.

Oevermann, U. (2000). Dienstleistung der Sozialbürokratie aus professionalisierungstheoretischer Sicht. In von Harrach, T. Eva-Marie von Loer \& O. Schmidtke (Hrsg.), Verwaltung des Sozialen. Formen der subjektiven Bewältigung eines Strukturkonflikts (S. 57-77). Konstanz: UVK.

Oevermann, U. (2013). Die Problematik der Strukturlogik des Arbeitsbündnisses und der Dynamik von Übertragung und Gegenübertragung in einer professionalisierten Praxis von Sozialarbeit. In R. Becker-Lenz, et al. (Hrsg.), Professionalität in der Sozialen Arbeit. Standpunkt, Kontroversen, Perspektiven (S. 119-149). Wiesbaden: Springer VS.

Ziegler, H. (2020). Das Elend mit dem Kindeswohl: Kindeswohlbezogener Kinderschutz als konservative Pädagogik. In H. Kelle \& S. Dahmen (Hrsg.), Ambivalenzen des Kinderschutzes (S. 172-188). Weinheim: Beltz Juventa. 